\title{
With a nasty organism, infective prosthetic endocarditis should not be dismissed
}

\author{
Farhang Yazdchi, MD, Tsuyoshi Kaneko, MD, and Sary Aranki, MD
}

\author{
From the Division of Cardiac Surgery, Department of Surgery, Brigham and Women's Hospital, Harvard Medical \\ School, Boston, Mass. \\ Disclosures: Authors have nothing to disclose with regard to commercial support. \\ Received for publication Jan 28, 2018; accepted for publication Jan 31, 2018. \\ Address for reprints: Sary Aranki, MD, Division of Cardiac Surgery, Brigham and Women's Hospital, 75 Francis \\ St, Boston, MA 02115 (E-mail: saranki@partners.org). \\ J Thorac Cardiovasc Surg 2018;155:2375-6 \\ $0022-5223 / \$ 36.00$ \\ Copyright (C) 2018 by The American Association for Thoracic Surgery \\ https://doi.org/10.1016/j.jtcvs.2018.01.066
}

In this issue of the Journal, Asadi and colleagues ${ }^{1}$ describe 2 cases of disseminated infection with Mycobacterium chimaera (MC) associated with a contaminated 3T heatercooler device (HCD) involving prosthetic graft and aortic valve material. The first patient developed symptoms 16 months after the original cardiac surgery. MC was isolated from a blood culture, urine specimen, and liver biopsy specimen, with evidence of ocular and spinal involvement. He eventually recovered after antibiotic therapy and surgery.

The second patient did not do as well. After reoperative surgery, the patient's postoperative course was complicated by fatigue, pancytopenia, and liver and renal dysfunction. He underwent a third reoperation and was found to have aortic root abscess and evidence of Mycobacterium avium intracellulare (MAI). Despite a prolonged course of antibiotics, the patient expired several months later.

These cases highlight 2 important points. First, timely diagnosis and effective medical management of recently described disseminated nontuberculous Mycobacterium infection (NTM) are key. The second point relates to surgical principal of treating infections involving prosthetic materials.

NTMs, which include MC and MAI, has been identified as the etiology of very serious infection in patients undergoing cardiac surgery with cardiopulmonary bypass with a particular type of HCD, where aerosolization of NTMcontaminated water tank and pipes occurs. The aerosolized particles travel with considerable horizontal airflow generated from the device exhaust, disrupting the vertical ceiling-to-floor operating room ultraclean laminar airflow and leading to surgical field contamination. ${ }^{2}$

NTMs are slow-growing with a long latent period, and symptoms are nonspecific. The interval between the procedure and onset of infection may be as long as 60 months. $^{3}$ Thus, diagnosis is difficult, especially when symptoms are nonspecific and an association with previous cardiac surgery is not established. Indeed, in the first patient, obtaining blood cultures for NTM was suggested by a relative of the patient

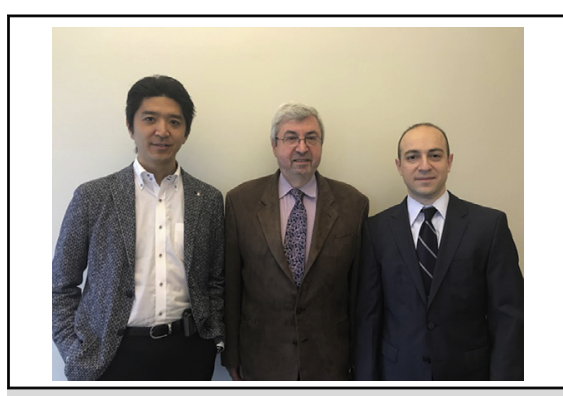

Tsuyoshi Kaneko, MD, Sary Aranki, MD, and Farhang Yazdchi, MD

\section{Central Message}

Timely diagnosis and effective management of disseminated Mycobacterium chimaera is extremely difficult. Aggressive surgical resection of prosthetic material, whether obviously infected or not, is warranted.

See Article page 2369.

who was aware of recent reports. Cultures take many weeks to identify the organism and establish susceptibility. Gene sequencing is usually required to identify the specific type of NTM, which may vary in invasiveness and susceptibility. Regardless, treatment requires combination antibiotic therapy, including the macrolides family. These antibiotics are bacteriostatic, and thus long-term treatment is required.

The basic surgical principle of treating infections involving prosthetic foreign material is complete removal and aggressive resection and debridement of infected tissue, such as abscesses, fistulas, and pseudo-aneurysms. In the first case, complete removal of previously placed prosthetic materials was performed along with complete debridement. The infection was eradicated, and the patient survived and returned to work within 4 months. In the second patient, incomplete resection of the aortic root abscess cavity at the time of the third sternotomy resulted in persistent enlargement of the abscess cavity as documented by subsequent imaging. He continued to have disseminated disease and died shortly thereafter. Even though cultures of the valve prosthesis were negative in the first patient, and unknown in the second, we are not sure that prosthetic valve endocarditis can be truly excluded in the setting of a very difficult-to-isolate organism.

In summary, a high degree of suspicion should always be maintained in the differential diagnosis in patients who underwent previous cardiac surgery and present with signs of a 
disseminated and nonspecific disease process. The key is to increase public health awareness and educate physicians who care for this patient population. ${ }^{4}$ Aggressive surgical resection and debridement, coupled with complete removal of all prosthetic material previously implanted, should be performed.

\section{References}

1. Asadi T, Mullin K, Roselli E, Johnston D, Tan CD, Rodriguez ER, et al. Disseminated Mycobacterium chimaera infection associated with heater-cooler units after aortic valve surgery without endocarditis. J Thorac Cardiovasc Surg. 2018;155:2369-74.

2. Sommerstein R, Rüegg C, Kohler P, Bloemberg G, Kuster SP, Sax H. Transmission of Mycobacterium chimaera from heater-cooler units during cardiac surgery despite an ultraclean air ventilation system. Emerg Infect Dis. 2016; 22:1008-13.

3. Allen KB, Yuh D, Schwartz SB, Lange R, Hopkins R, Kelly B, et al. Nontuberculous Mycobacterium infections associated with heater-cooler devices. Ann Thorac Surg. 2017;104:1237-42.

4. Centers for Disease Control and Prevention. Interim guide for the identification of possible cases of nontuberculous Mycobacterium infections associated with exposure to heater-cooler units. Available at: https://www.cdc.gov/hai/pdfs/outbreaks/ Guide-for-Case-Finding.pdf. Accessed February 19, 2018. 